\title{
El tiotropio disminuyó exacerbaciones pero no la declinación de la función pulmonar en pacientes con enfermedad pulmonar obstructiva crónica
}

Tiotropium reduced exacerbations but no decline in lung function in patients with chronic obstructive pulmonary disease

Tashkin D y col. N Engl J Med. 2008 359(15):1543-54

\section{Objetivo}

Evaluar la eficacia del tiotropio en comparación con placebo en la mejoría de la declinación funcional en pacientes con enfermedad pulmonar obstructiva crónica (EPOC).

\section{Diseño, lugar y pacientes}

Ensayo clínico controlado, aleatorizado y doble ciego, realizado en 37 países, con cuatro años de seguimiento. Participaron 5.993 pacientes mayores de 40 años (edad promedio 65 años, $75 \%$ varones) con EPOC e historia de tabaquismo de diez o más paquetes/año, y volumen espiratorio forzado en el primer segundo (VEF1) menor ó igual a 70\% luego del uso de broncodilatadores.

\section{Intervención}

18 microgramos de tiotropio (grupo intervención) contra placebo (grupo control). Se permitió el uso concomitante de otras medicaciones respiratorias, salvo otros anticolinérgicos inhalatorios.

Medición de resultados principales

Los pacientes fueron sometidos a monitoreo clínico (cada tres meses) y espirométrico (cada seis). El resultado primario fue la tasa promedio de declinación en el VEF1 antes y después del uso de broncodilatadores (cambio de la pendiente de deterioro). Secundariamente se evaluó la calidad de vida, el número de exacerbaciones e internaciones relacionadas a la EPOC, así como la mortalidad general y específica.

\section{Resultados}

Al menos un 73 y $60 \%$ de los pacientes completaron el seguimiento a los 2 y 3,7 años, respectivamente. No se observaron diferencias estadísticamente significativas en la declinación del deterioro de los parámetros de función respiratoria, ni de calidad de vida. Aunque las mediciones estáticas de puntuación de este último parámetro otorgaron cierto beneficio al tratamiento con tiotropio, la diferencia lograda en ningún momento alcanzo el umbral de significancia clínica. El tratamiento con tiotropio logro reducir la frecuencia y duración de las exacerbaciones (se necesitaría agregar tiotropio al tratamiento de base en ocho pacientes para evitar una exacerbación adicional por año, y a 65 para reducir, además, un día de la duración anual de las exacerbaciones por paciente tratado); pero no tuvo efecto sobre el número de internaciones ni sobre la mortalidad. La aparición de efectos adversos fue mas frecuente en el grupo placebo.

Tabla 1: tasas anuales de declinación del volumen espiratorio forzado en el primer segundo (VEF1) del puntaje de calidad de vida y del número de exacerbaciones.

\begin{tabular}{|c|c|c|c|c|c|}
\hline & & Tiotropio & Placebo & RRA (IC95\%) & NNT \\
\hline \multirow{2}{*}{$\begin{array}{l}\text { Volumen espiratorio forzado en el } \\
\text { primer segundo ( } \mathrm{mL} / \mathrm{año} \text { ) }\end{array}$} & Pre-broncodilatación & 30 & 30 & $0(-4$ a 4$)$ & - \\
\hline & Post-broncodilatación & 40 & 42 & $-2(-6$ a 2) & \\
\hline \multicolumn{2}{|l|}{ Calidad de vida ${ }^{a}$} & 1,25 & 1,21 & $0,04(-0,2$ a 0,3$)$ & \\
\hline & & Tiotropio & Placebo & RR (IC95\%) & - \\
\hline \multirow{2}{*}{$\begin{array}{l}\text { Exacerbaciones } \\
\text { (por paciente por año) }\end{array}$} & Episodios & 0,73 & 0,85 & $0,86(0,81$ a 0,91$)$ & 8,3 \\
\hline & Días & 12,11 & 13,64 & $0,89(0,83$ a 0,95$)$ & 65,4 \\
\hline \multicolumn{2}{|l|}{ Internaciónes (por paciente por año) } & 0,15 & 0,16 & $0,94(0,82$ a 1,07$)$ & - \\
\hline
\end{tabular}

aPuntaje de St, Gregory (con un rango de 0 a 100, las valores mas altos indican deterioro, considerándose clínicamente significativo un cambio mayor a cuatro puntos).

\section{Conclusiones}

La adición de $18 \mathrm{mcg} /$ día de tiotropio durante cuatro años no mejora la declinación de la función pulmonar, la calidad de vida, la tasa de internaciones, ni la mortalidad general o especí- fica; pero si demostró beneficios en la reducción del número y la duración de las exacerbaciones.

Palabras claves: Enfermedad Pulmonar Obstructiva Crónica, Tiotropio, VEF Key words: Chronic Obstructive Pulmonary Disease, Tiotropium, FEV'. Fuente de financiamiento: Boehringer Ingelheim y Pfizer.

\section{Comentario}

Por lo previamente explicitado podemos afirmar que si bien el agregado de tiotropio mejora la frecuencia de reagudizaciones relacionadas al EPOC, no logra alterar su progresión natural. En este sentido habría sido interesante haber contado en este ensayo clínico con una rama adicional que hubiera comparado la eficacia de tiotropio con bromuro de ipratropio (también anticolinérgico) y quizá también su costo-efectividad y adherencia, dado que el tiotropio es aproximadamente diez veces más costoso, pero tiene la ventaja de requerir una única aplicación diaria. Por ejemplo, cuanto dinero más habría destinar para que un paciente tenga un día menos de exacerbación de su EPOC por año. Por otro lado, cabe mencionar que este estudio aclara la controversia existente en relación a la seguridad de la medicación anticolinérgica en pacientes con EPOC. Sus resultados son contrarios a los reportados en una revisión sistemática realizada previamente ${ }^{1}$ donde se reportaba un aumento del riesgo de infarto agudo de miocardio y de morta-lidad cardiovascular.

\section{Conclusiones de los comentadores}

El tiotropio proporcionó un efecto modesto en algunos de los resultados secundarios. Teniendo en cuenta su costo, seria apropiado revisar la mejor evidencia disponible que lo compare con el bromuro de ipratropio para decidir racionalmente su uso.

Cynthia Dreksler y Gabriel Villalon [ Servicio Medicina Familiar y Comunitaria del Hospital Italiano de Buenos Aires. cynthia.dreksler@hospitalitaliano.org.ar ]

Recibido el 10/06/2009 y aceptado el 11/07/2009

Yamauchi G, Tiotropio disminuyó exacerbaciones pero no declinación de la función pulmonar en pacientes con EPOC, Evid Act Pract Ambul, 12(3), 94, Jul-Sep, 2009, Comentado de: Tashkin D y col. A 4-year trial of tiotropium in chronic obstructive pulmonary disease, N Engl J Med, 2008 Oct 9;359(15):1543-54, PMID: 18836213, Disponible en URL: http://content,nejm,org/cgi/reprint/359/15/1543,pdf (último acceso 16/08/2009).

Referencia

1.Singh S, Loke YK, Furberg CD, Inhaled anticholinergics and risk of major adverse cardiovascular events in patients with chronic obstructive pulmonary disease: a systematic review and meta-analysis, JAMA, 2008 Sep 24;300(12):1439-50. 